\title{
Effects of melatonin on cognitive impairment and hippocampal neuronal damage in a rat model of chronic cerebral hypoperfusion
}

\author{
CHOONG HYUN LEE ${ }^{1}$, JOON HA PARK ${ }^{2}$, JI HYEON AHN ${ }^{2}$ and MOO-HO WON ${ }^{2}$ \\ ${ }^{1}$ Department of Pharmacy, College of Pharmacy, Dankook University, Cheonan, Chungcheongnam 330-714; \\ ${ }^{2}$ Department of Neurobiology, School of Medicine, Kangwon National University, \\ Chuncheon, Gangwon 200-701, Republic of Korea
}

Received January 15, 2015; Accepted October 9, 2015

DOI: $10.3892 /$ etm.2016.3216

\begin{abstract}
Chronic cerebral hypoperfusion $(\mathrm{CCH})$, which induces oxidative stress and inflammation in the brain, has previously been associated with cognitive impairment and neuronal cell damage. Melatonin is a well-known free radical scavenger and antioxidant; therefore, the present study investigated the protective effects of melatonin against $\mathrm{CCH}$-induced cognitive impairment and neuronal cell death in a $\mathrm{CCH}$ rat model, which was generated via permanent bilateral common carotid artery occlusion (2VO). The rats in the $2 \mathrm{VO}$ group exhibited markedly increased escape latencies in a Morris water maze test, as compared with the rats in the sham group. In addition, increased neuronal cell damage was detected in the hippocampal CA1 region of the $2 \mathrm{VO}$ rats, as compared with the rats in the sham group. Treatment of the $2 \mathrm{VO}$ rats with melatonin significantly reduced the escape latency and neuronal cell damage, and was associated with reduced levels of malondialdehyde, microglial activation, and tumor necrosis factor- $\alpha$ and interleukin- $1 \beta$ in the ischemic hippocampus. The results of the present study suggest that melatonin may attenuate $\mathrm{CCH}$-induced cognitive impairment and hippocampal neuronal cell damage by decreasing oxidative stress, microglial activation and the production of pro-inflammatory cytokines in the ischemic hippocampus.
\end{abstract}

\section{Introduction}

Chronic cerebral hypoperfusion $(\mathrm{CCH})$, which has previously been associated with the onset of vascular dementia and cognitive dysfunction $(1,2)$, induces oxidative stress and

Correspondence to: Professor Moo-Ho Won, Department of Neurobiology, School of Medicine, Kangwon National University, 192-1 Hyoja-2-dong, Chuncheon, Gangwon 200-701, Republic of Korea

E-mail:mhwon@kangwon.ac.kr

Key words: melatonin, chronic cerebral hypoperfusion, spatial learning and memory, neuronal damage, microglial activation, pro-inflammatory cytokines inflammatory reactions that may contribute to cognitive impairment and neuronal damage in various regions of the brain, including the hippocampus and cerebral cortex (1,3-7). Previous reports of $\mathrm{CCH}$-induced neuropathological alterations have focused on the hippocampus, likely due to its critical involvement in learning and memory (6-9). In addition, the hippocampal CA1 region was shown to be vulnerable to the effects of $\mathrm{CCH}$ and cerebral ischemia (6-11). A rat model of permanent bilateral common carotid artery occlusion (2VO) is widely used to investigate $\mathrm{CCH}$ and $\mathrm{CCH}$-related vascular dementia, as the $2 \mathrm{VO}$ procedure results in a significant reduction in cerebral blood flow $(4,6,7,9)$. Furthermore, previous studies have suggested that an association exists between 2VO-induced cognitive impairment and hippocampal damage $(1,4,7,12,13)$.

Melatonin (N-acetyl-5-methoxytryptamine), which is a well-known and potent free radical scavenger and antioxidant, sequesters hydroxyl radicals and stimulates antioxidative enzymes $(14,15)$. Furthermore, melatonin has been reported to exert neuroprotective effects in various animal models of neurodegenerative diseases $(16,17)$, including reducing the infarct volume and neurological deficit in an animal model of focal cerebral ischemia, and attenuating hippocampal CA1 region pyramidal neuronal cell death/damage in various animal models of global cerebral ischemia $(10,18,19)$. The neuroprotective effects of melatonin have previously been associated with decreased oxidative stress and neuroinflammation $(20,21)$.

Ozacmak et al (6) reported that melatonin was able to protect hippocampal neurons against the effects of $\mathrm{CCH}$ by reducing oxidative stress and heat shock protein 70 expression levels in an ovariectomized rat; however, the underlying mechanisms by which melatonin may protect against $\mathrm{CCH}$-induced cognitive impairment and neuropathological alterations are yet to be fully elucidated. The present study aimed to investigate the neuroprotective effects of melatonin against $\mathrm{CCH}$-induced cognitive impairment and neuronal damage/death in a rat model of 2VO-induced $\mathrm{CCH}$.

\section{Materials and methods}

Experimental rats. A total of 48 male Sprague-Dawley rats (age, 16 weeks; weight, $350 \pm 20 \mathrm{~g}$ ) were obtained from 
RaonBio Inc. (Yongin, South Korea). The rats were housed in individual cages (temperature, $23^{\circ} \mathrm{C}$; humidity, $60 \%$ ) under a $12 \mathrm{~h}$ light/dark cycle, and provided with ad libitum access to water and commercial chow throughout the experimental period. The rat handling and care procedures adhered to guidelines that are in compliance with current international laws and policies (22), and the experimental protocol was approved by the Institutional Animal Care and Use Committee of Kangwon National University (Chuncheon, South Korea). All experiments performed were designed to minimize the number of rats used and their suffering.

Experimental groups and melatonin treatment. In order to investigate the effects of melatonin (Sigma-Aldrich, St. Louis, MO, USA) on $\mathrm{CCH}$-induced cognitive impairment and hippocampal neuronal damage, the rats were designated into three groups ( $n=16 /$ group), as follows: i) A vehicle-treated ( $1 \%$ ethanol in saline), sham-operated group (sham-group); ii) a vehicle-treated, 2VO-operated group (2VO-group); and iii) a $10 \mathrm{mg} / \mathrm{kg}$ melatonin-treated, 2VO-operated group (mel-2VO-group). Melatonin, dissolved in ethanol and saline (final concentration, 1\%), was intraperitoneally administered once daily, between days 1 (surgery) and 28 (sacrifice). The dose of melatonin administered to the rats was selected on the basis of previous studies $(6,10)$.

Production of a rat $\mathrm{CCH}$ model. A rat model of $\mathrm{CCH}$ was generated via $2 \mathrm{VO}$, as outlined in previous studies $(4,6,7)$. Briefly, the rats were anesthetized using a mixture of $2.5 \%$ isoflurane (Baxter, Deerfield, IL, USA), 33\% oxygen and $67 \%$ nitrous oxide. A midline cervical incision was used to expose the bilateral common carotid arteries and to separate them from the vagus nerve. In the 2VO-group and mel-2VO-group rats, the bilateral common carotid arteries were ligated using a 5-0 silk suture. The body temperatures of the rats under free-regulating or normothermic $\left(37 \pm 0.5^{\circ} \mathrm{C}\right)$ conditions were monitored using rectal temperature probes (TR-100; Fine Science Tools, Foster City, CA, USA), and were maintained using a thermometric blanket prior to, during and following surgery, until the rats had recovered from the anesthesia. Thereafter, the animals were maintained in a thermal incubator (Mirae Medical Industry, Seoul, Korea), prior to sacrifice. The sham-group rats were subjected to an identical surgical procedure; however, the bilateral common carotid arteries were not occluded. All rats that did not survive the surgery were replaced.

Morris water maze test. The spatial learning and memory functions of all rats were evaluated using a Morris water maze test, as outlined in previous studies $(4,7,8,23)$. Briefly, the maze consisted of a black circular tank (diameter, $120 \mathrm{~cm}$; height, $50 \mathrm{~cm}$ ) filled with water (temperature, $23^{\circ} \mathrm{C}$; depth, $40 \mathrm{~cm}$ ). A black platform was submerged $1.5 \mathrm{~cm}$ beneath the water surface throughout the duration of the test. The rats were allowed a maximum of $120 \mathrm{sec}$ to locate the platform, during which the escape latencies and swimming paths onto the platform were recorded using a video camera linked to a computer via using the SMART video tracking system (Panlab, Barcelona, Spain). All rats were subjected to four trials per day for five consecutive days (between days 23 and 27 following surgery).
Tissue processing and neuronal damage. The rats ( $n=6 /$ group) were anesthetized using 30\% chloral hydrate (10 ml/kg; Sigma-Aldrich), 28 days following surgery, after which they were treated with $0.1 \mathrm{M}$ phosphate-buffered saline (PBS; pH 7.4), and then $4 \%$ paraformaldehyde in $0.1 \mathrm{M}$ phosphate-buffer ( $\mathrm{pH} 7.4$ ), via a transcardial perfusion. Subsequently, the brains were removed and postfixed in $4 \%$ paraformaldehyde for $6 \mathrm{~h}$, after which the brain tissues were cryoprotected via incubation with $30 \%$ sucrose overnight. Thereafter, frozen tissue samples were serially sectioned using a cryostat (Leica Microsystems GmbH, Wetzlar, Germany) into $30 \mu \mathrm{m}$ coronal sections, which were subsequently distributed into 6-well plates containing PBS.

In order to examine neuronal damage and the neuroprotective effects of melatonin in the hippocampal CA1 region, Neuronal Nuclei (NeuN) immunohistochemistry and Fluoro-Jade B (F-J B) histofluorescence staining were conducted, as outlined in previous studies $(10,23,24)$. Briefly, the tissue sections were treated with $0.3 \%$ hydrogen peroxide in PBS for $30 \mathrm{~min}$, followed by $10 \%$ normal goat serum (Vector Laboratories, Inc., Burlingame, CA, USA) in 0.05 M PBS for $30 \mathrm{~min}$. Subsequently, the tissue sections were incubated with diluted mouse anti-NeuN (cat. no. MAB377; dilution, 1:1,000; Chemicon International, Inc., Temecula, CA, USA) overnight at $4^{\circ} \mathrm{C}$, after which they were incubated with streptavidin peroxidase-conjugated biotinylated goat anti-mouse immunoglobulin G (cat. no. BA-9200-1.5; dilution, 1:200; Vector Laboratories, Inc.). For visualization, the tissue sections were stained with 3,3'-diaminobenzidine (Sigma-Aldrich) in $0.1 \mathrm{M}$ Tris-HCl buffer ( $\mathrm{pH} 7.2$ ), and mounted on gelatin-coated slides. Following dehydration, the tissue sections were mounted with Canada balsam (Kanto Chemical, Co., Inc., Tokyo, Japan).

For F-J B histofluorescence staining, the tissue sections were incubated in a $1 \%$ sodium hydroxide solution, supplemented with first $80 \%$, and then $70 \%$ ethanol. Subsequently, the sections were treated with $0.06 \%$ potassium permanganate, followed by staining with $0.0004 \%$ F-J B staining solution (Histo-Chem, Inc., Jefferson, AR, USA). After washing, the sections were mounted on a slide warmer (temperature, $\sim 50^{\circ} \mathrm{C}$ ), and were examined using an epifluorescent microscope (Carl Zeiss AG, Oberkochen, Germany), with a blue (450-490 nm) excitation light and a barrier filter. Neurons undergoing degeneration exhibited bright fluorescence, as compared with the background.

In order to evaluate the neuroprotective effects of melatonin, NeuN-immunoreactive neurons and F-J B-positive cells in the hippocampal CA1 region were counted (magnification, 40x) and digital images were captured using an Axio Imager 2 light microscope (Carl Zeiss AG), equipped with a digital camera (Axiocam; Carl Zeiss AG) connected to a PC monitor. Six coronal sections with $150 \mu \mathrm{m}$ intervals were selected for each rat, and cell counts were obtained by averaging the counts from each rat.

Measurement of lipid peroxidation. In order to examine the effects of melatonin on the rates of lipid peroxidation in the hippocampus of the $\mathrm{CCH}$ rats, malondialdehyde (MDA) formation was measured using the Bioxytech ${ }^{\circledR}$ MDA-586 kit (OxisResearch, Portland, OR, USA), according to our previous study (23). Briefly, the rats ( $\mathrm{n}=5 /$ group) were sacrificed 28 days 
following surgery, after which the hippocampus was removed and homogenized in $20 \mathrm{mM}$ PBS (pH 7.4) containing $5 \mathrm{mM}$ butylated hydroxytoluene, using a Vibra-Cell ultrasonic processor (Sonics and Materials, Inc., Newtown, CT, USA). Homogenates were centrifuged at $3,000 \mathrm{x}$ g for $10 \mathrm{~min}$ at $4^{\circ} \mathrm{C}$, and the supernatants were collected for MDA quantification. Probucol $(10 \mu \mathrm{l})$ and diluted R1 reagent $(640 \mu \mathrm{l} ; 1: 3$ of methanol:N-methyl-2-phenylindole) were added to the supernatants and subsequently mixed with $150 \mu 112 \mathrm{~N}$ hydrochloric acid. Each reaction was incubated for $60 \mathrm{~min}$ at $45^{\circ} \mathrm{C}$, after which the supernatants were centrifuged at $10,000 \mathrm{x} \mathrm{g}$ for $10 \mathrm{~min}$. Absorbance at $586 \mathrm{~nm}$ was measured using a BioTek ELx800 absorbance microplate reader (Bio-Tek Instruments Inc., Winooski, VT, USA) as an indication of MDA content.

Microglial activation. In order to investigate microglial activation, immunohistochemical staining using rabbit anti-ionized calcium-binding adapter molecule-1 (Iba-1) antibodies (cat. no. 019-19741; 1:200; Wako Pure Chemical Industries, Ltd., Osaka, Japan) was performed, as outlined in a previous study (25). The density of Iba-1-immunoreactive structures was evaluated on the basis of optical density (OD), which was calculated following the transformation of the mean gray level with ImageJ version 1.42 software (imagej.nih.gov/ij) using the formula: OD=log (256/mean gray level). The OD of the background was subtracted from areas adjacent to the measured area. After the background density was subtracted, a ratio of the OD of the image file was calibrated as a percentage (relative optical density, ROD) using Adobe Photoshop version 8.0 (Adobe Systems, San Jose, CA, USA), and then analyzed using ImageJ software. A ratio of the ROD was calibrated as $\%$, with the sham-group designated as $100 \%$.

Quantification of the protein expression levels of pro-inflammatory cytokines. In order to examine the effects of melatonin on the protein expression levels of specific pro-inflammatory cytokines in the hippocampus of the $\mathrm{CCH}$ rat model, enzyme linked immunosorbent assays (ELISA) for tumor necrosis factor (TNF)- $\alpha$ and interleukin (IL)- $1 \beta$ were performed, in accordance with a previous study (26). Briefly, the rats ( $n=5 /$ group) were sacrificed 28 days following surgery, and the hippocampi were removed and homogenized using a Vibra-Cell ultrasonic processor. After centrifugation of the homogenates at 14,000 $\mathrm{x} \mathrm{g}$ for $20 \mathrm{~min}$ at $4^{\circ} \mathrm{C}$, the supernatant was collected. The levels of TNF- $\alpha$ and IL-1 $\beta$ were examined using a commercial Invitrogen ELISA kit (Thermo Fisher Scientific, Inc., Waltham, MA, USA), according to the manufacturer's protocol.

Statistical analysis. The data are presented as the mean \pm standard error of the mean. Statistical differences were analyzed using two-way analysis of variance followed by Dunnett's test, using SPSS 12.0 software (SPSS, Inc., Chicago, IL, USA). $\mathrm{P}<0.05$ was considered to indicate a statistically significant difference.

\section{Results}

Morris water maze test. The spatial learning and memory functions of all rats were examined using the Morris water

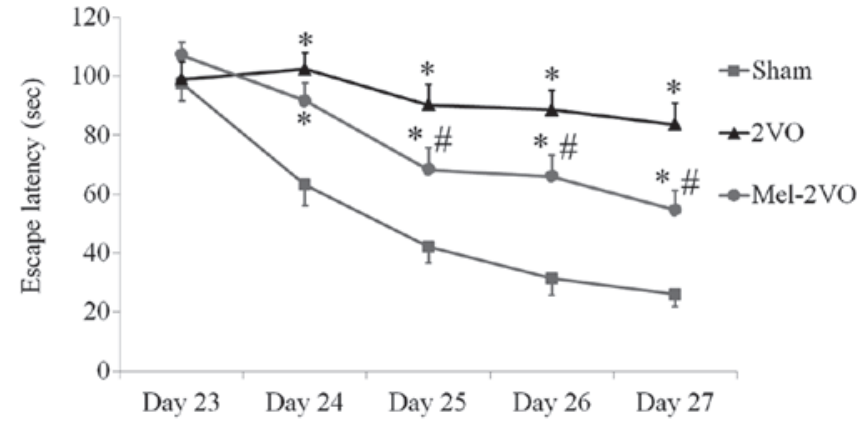

Figure 1. Escape latencies of the rats in the sham-, 2VO- and mel-2VO-groups on days 23 to 27 following the $2 \mathrm{VO}$ surgery ( $\mathrm{n}=14 /$ group), as determined by Morris water maze test. Data are presented as the mean \pm standard error of the mean. ${ }^{*} \mathrm{P}<0.05$ vs. the sham-group. ${ }^{\#} \mathrm{P}<0.05$ vs. the $2 \mathrm{VO}$-group. $2 \mathrm{VO}$, bilateral common carotid artery occlusion-operated group; mel, melatonin.

maze test, which was conducted four times per day between days 23 and 27 following surgery. The escape latencies for the rats in the sham-group markedly decreased over the five days, whereas the escape latencies for the rats in the 2VO-group were significantly longer, as compared with the rats in the sham-group, and did not significantly decrease over the trial period ( $\mathrm{P}<0.05$, Fig. 1). Conversely, the escape latencies of the rats in the mel-2VO-group significantly decreased from day 3 in the trial period, as compared with that in the 2VO-group rats $(\mathrm{P}<0.05$, Fig. 1). However, the rats in the mel-2VO-group had markedly longer escape latencies, as compared with the rats in the sham-group (Fig. 1).

Neuroprotective effects of melatonin. $\mathrm{CCH}$-induced neuronal cell damage and the neuroprotective effects of melatonin in the hippocampal CA1 region were examined 28 days following surgery using NeuN immunohistochemistry and F-J B histofluorescence staining (Fig. 2). High numbers of NeuN-immunoreactive $(+)$ cells were detected in the CA1 region of the sham-group rats, whereas no F-J B-positive $(+)$ cells were detected in this region (Fig. 2A, D and G). Conversely, in the 2VO-group rats, the number of NeuN+ cells was significantly reduced, and the number of F-J B+ cells were markedly increased in the stratum pyramidale of the hippocampal CA1 region (Fig. 2B, E and G). Elevated numbers of $\mathrm{NeuN}+$ cells were detected in the mel-2VO-group rats: $73.7 \%$ of the pyramidal cells were protected, as compared with that in the sham-group (Fig. 2C and G). In addition, the number of F-J B+ cells in the mel-2VO-group was significantly reduced, as compared with the rats in the 2VO-group (Fig. $2 \mathrm{~F}$ and $\mathrm{G}$ ).

Quantification of MDA levels in the rat hippocampus. In order to investigate the effects of melatonin on $\mathrm{CCH}$-induced lipid peroxidation, MDA levels were measured in the hippocampal samples of all rats. Hippocampal MDA levels in the sham-group were $1.83 \pm 0.16 \mathrm{nmol} / \mathrm{mg}$ protein, and were significantly increased (2.6-fold; $\mathrm{P}<0.05$ ) in the 2VO-group rats (Fig. 3). However, hippocampal MDA levels in the mel-2VO-group were significantly reduced (60\% of $2 \mathrm{VO}$-group levels), as compared with the 2VO-group $(\mathrm{P}<0.05$, Fig. 3$)$.

Microglial activation. Iba-1 immunohistochemistry in the hippocampal CA1 region (Fig. 4) demonstrated that, in the 

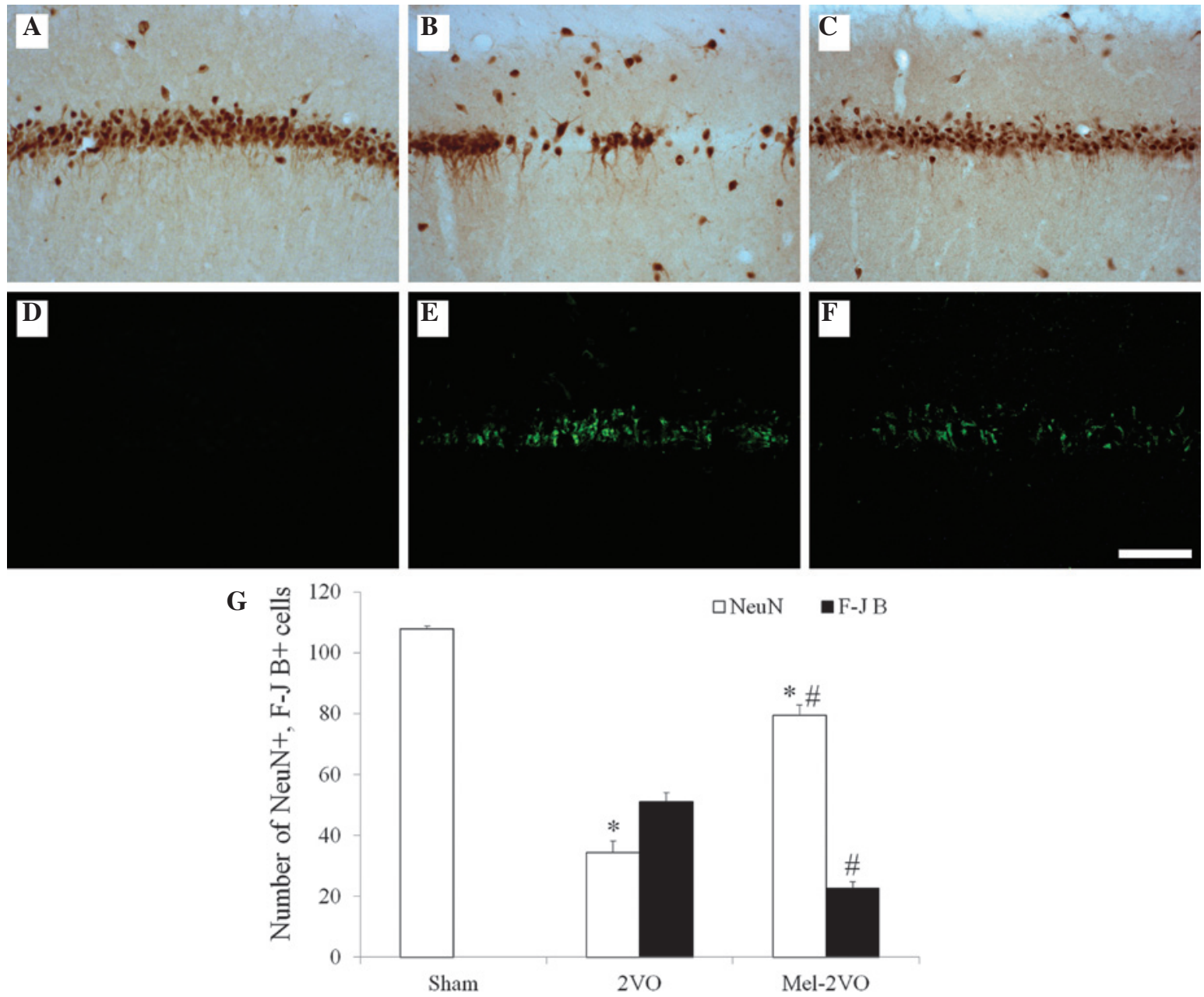

Figure 2. (A-C) NeuN immunohistochemistry and (D-F) F-J B histofluorescence staining in the hippocampal CA1 region of the (A and D) sham(B and E) 2VO- and (C and F) mel-2VO- groups. NeuN+ neurons significantly decreased, whereas F-J B+ cells (arrows) markedly increased, in number in the SP of the 2VO-group rats. Conversely, NeuN+ neurons significantly increased, and F-J B+ cells significantly decreased, in number in the SP of the mel-2VO-group rats, as compared with the 2VO-group rats (scale bar $=100 \mu \mathrm{m}$ ). (G) Analysis of the number of NeuN+ neurons and F-J B+ cells in the CA1 region of the sham-, $2 \mathrm{VO}$ - and mel-2VO-group rats. Data are presented as the mean \pm standard error of the mean. " $\mathrm{P}<0.05$ vs. the sham-group, ${ }^{\sharp} \mathrm{P}<0.05$ vs. the 2VO-group. SO, stratum oriens; SR; stratum radiatum; SP, stratum pyramidale; NeuN, Neuronal Nuclei; F-J B, Fluoro-Jade B; 2VO, bilateral common carotid arteries occlusion-operated; mel, melatonin.

sham-group, ramified Iba-1+ microglia containing a small cytoplasm, known as resting microglia, were distributed throughout the hippocampal CA1 region (Fig. 4A). In the CA1 region of the 2VO-group rats, Iba-1 immunoreactivity significantly increased, as compared with the sham group rats $(\mathrm{P}<0.05$, Fig. 4B and D). In addition, numerous Iba- $1+$ microglia exhibited hypertrophy and activation, with round forms, and were aggregated in the stratum pyramidale of the CA1 region of the 2VO-group rats (Fig. 4B). Following treatment with melatonin, Iba-1 immunoreactivity in the mel-2VO-group significantly decreased, as compared with the 2VO-group ( $\mathrm{P}<0.05$, Fig. $4 \mathrm{C}$ and $\mathrm{D})$; however, a higher number of microglia exhibited activation in the mel-2VO-group rats, as compared with the sham-group rats.

Quantification of TNF- $\alpha$ and IL-1 $\beta$ protein expression levels. The TNF- $\alpha$ and IL-1 $\beta$ hippocampal protein expression levels in the sham-group rats were $24.44 \pm 1.65 \mathrm{pg} / \mathrm{mg}$ and $13.95 \pm 0.92 \mathrm{pg} / \mathrm{mg}$ protein, respectively. In the $2 \mathrm{VO}$-group, protein expression levels of TNF- $\alpha$ and IL-1 $\beta$ were significantly increased, as compared with those in the sham-group

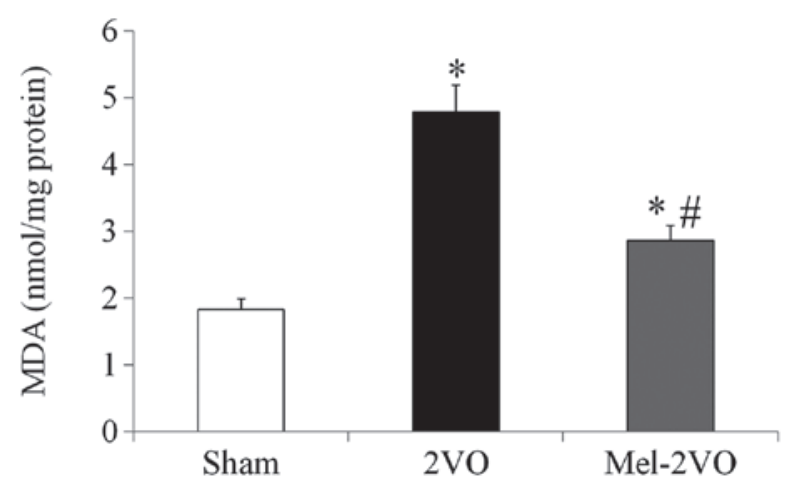

Figure 3. Analysis of hippocampal MDA levels in the sham-, 2VO- and mel-2VO-groups. Data are presented as the mean \pm standard error of the mean. ${ }^{~} \mathrm{P}<0.05$ vs. the sham-group, ${ }^{\#} \mathrm{P}<0.05$ vs. the $2 \mathrm{VO}$-group. MDA, malondialdehyde, $2 \mathrm{VO}$, bilateral common carotid artery occlusion-operated; mel, melatonin.

rats $(\mathrm{P}<0.05$, Fig. 5). Conversely the TNF- $\alpha(31.11 \pm 2.85 \mathrm{pg} / \mathrm{mg})$ and IL-1 $\beta(18.67 \pm 2.05 \mathrm{pg} / \mathrm{mg})$ protein expression levels were significantly decreased in the mel-2VO-group rats, as compared 

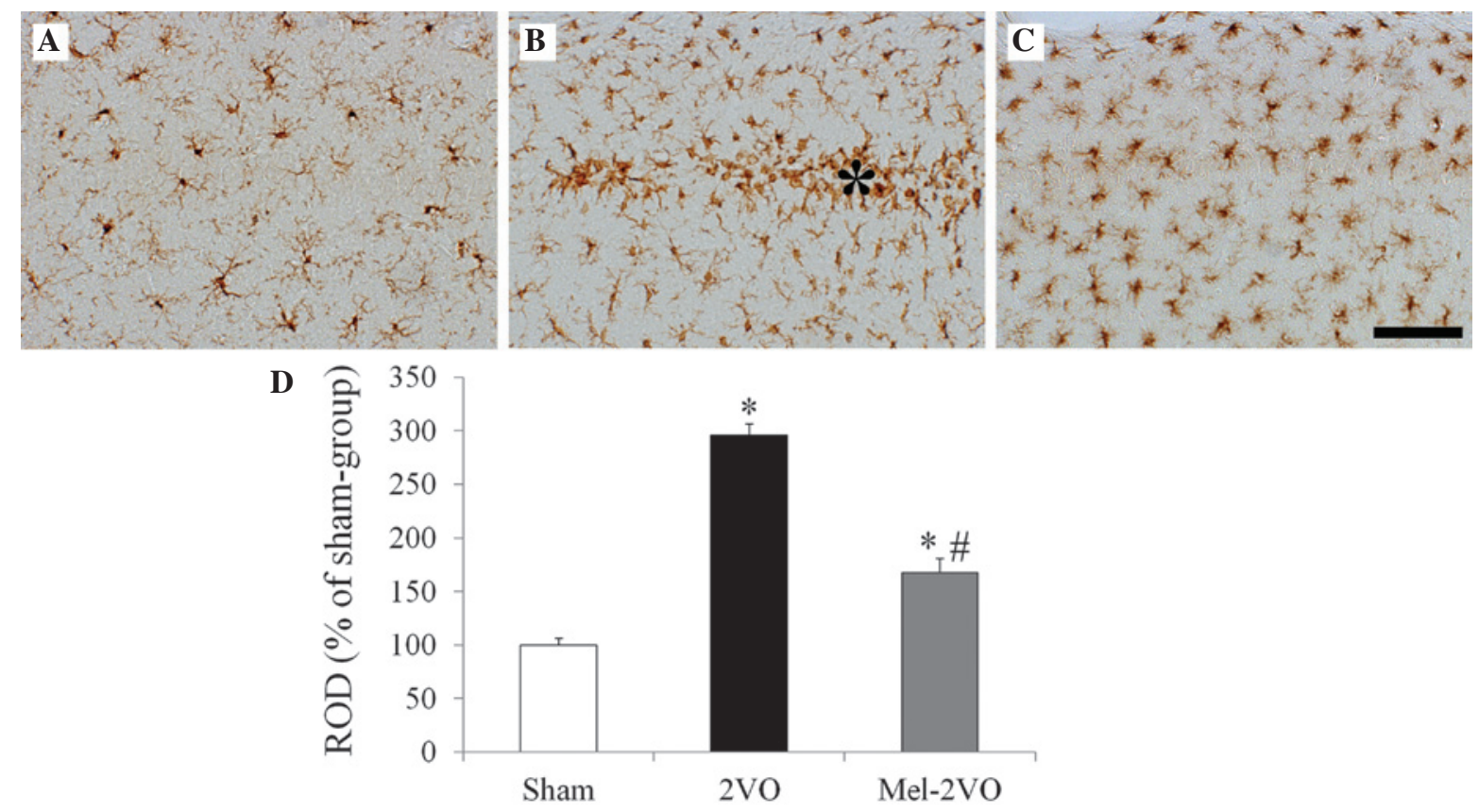

Figure 4. Iba-1 immunohistochemistry in the hippocampal CA1 region of the (A) sham-, (B) 2VO- and (C) mel-2VO- groups. In the 2VO-group, Iba-1 immunoreactivity was markedly increased, and the activated Iba-1+ microglia aggregated in the SP (asterisk). In the mel-2VO-group, Iba-1 immunoreactivity was significantly decreased, as compared with the $2 \mathrm{VO}$-group rats (scale bar=50 $\mu \mathrm{m}$ ). (D) ROD was calculated as the percentage of Iba- $1+$ structures in the $\mathrm{CA} 1$ region of the sham-, 2VO- and mel-2VO-group rats. Data are presented as the mean \pm standard error of the mean. Asterisk indicates SP. "P $<0.05$ vs. the sham-group, ${ }^{\prime} \mathrm{P}<0.05$ vs. the $2 \mathrm{VO}$-group. Iba-1, ionized calcium-binding adapter molecule-1; 2VO, bilateral common carotid arteries occlusion-operated; ROD, relative optical density.

A

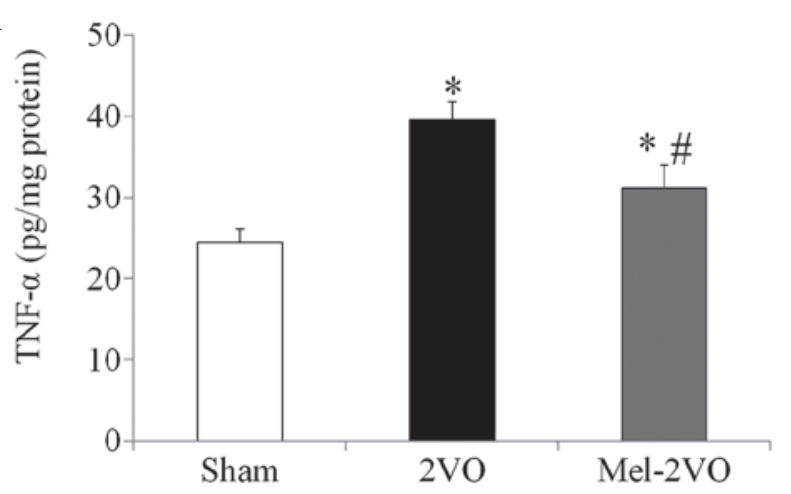

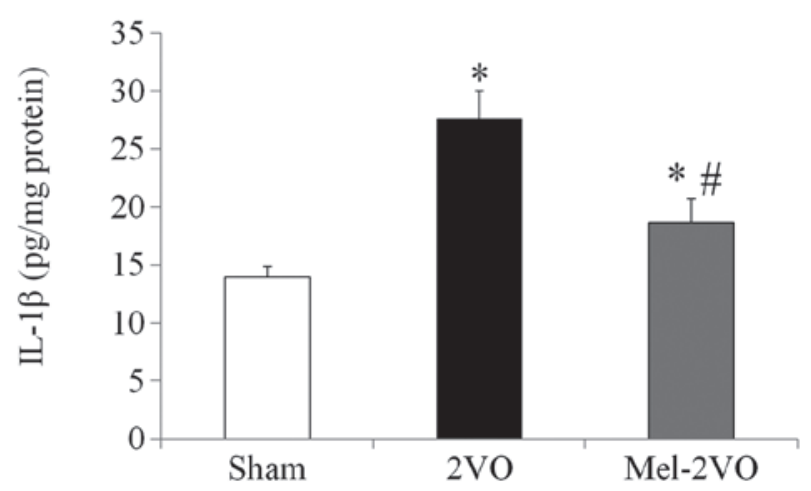

Figure 5. (A) TNF- $\alpha$ and (B) IL-1 $\beta$ hippocampal protein expression levels in the sham-, 2VO- and mel-2VO-groups. Data are presented as the mean \pm standard error of the mean. ${ }^{*} \mathrm{P}<0.05$ vs. the sham-group, ${ }^{\mathrm{P}} \mathrm{P}<0.05$ vs. the $2 \mathrm{VO}$-group. TNF, tumor necrosis factor; IL, interleukin; $2 \mathrm{VO}$, bilateral common carotid arteries occlusion-operated; mel, melatonin.

with those in the 2VO-group rats $(\mathrm{P}<0.05$, Fig. 5); however, TNF- $\alpha$ and IL-1 $\beta$ protein expression levels were significantly higher than those in the sham-group rats $(\mathrm{P}<0.05$, Fig. 5).

\section{Discussion}

Previous studies have detected hippocampus-dependent spatial learning and memory impairment in rat models of $2 \mathrm{VO}(1,4,7-9,12,13,27)$. The present study examined the effects of melatonin on $\mathrm{CCH}$-induced cognitive impairment, using the Morris water maze test, and demonstrated that rats in the 2VO-group had significantly longer escape latencies, as compared with the rats in the sham-group. In addition, escape latencies of the rats in the mel-2VO-group were significantly decreased, as compared with the $2 \mathrm{VO}$-group rats. To the best of our knowledge, the present study is the first to report the effects of melatonin on $\mathrm{CCH}$-induced cognitive impairment, and demonstrated that melatonin treatment was able to improve $\mathrm{CCH}$-induced hippocampus-dependent spatial learning and memory impairment. These results are consistent with those of a previous study, which reported that post-ischemic melatonin treatment in a rat model of global cerebral ischemia reduced escape latencies, as compared with a sham-group (19).

Previous studies have detected significant reductions in the number of pyramidal cells in the hippocampal CA1 region following $2 \mathrm{VO}$, and therefore suggested that $2 \mathrm{VO}$-induced cognitive impairment may be associated with a loss of hippocampal CA1 cells $(1,7,12,13,27)$. In the present study, a 
significant reduction in the number of $\mathrm{NeuN}+$ neurons, and a marked increase in the number of F-J B+ (a marker for degenerating neurons) cells, were detected in the stratum pyramidale of the hippocampal CA1 region in the 2VO-group rats. In addition, the ability of melatonin to protect against $\mathrm{CCH}$-induced neuronal cell death in the hippocampal CA1 region was investigated using NeuN immunohistochemistry and F-J B histofluorescence staining. Melatonin treatment significantly reduced $\mathrm{CCH}$-induced neuronal cell death in the hippocampal CA1 region of 2VO-group rats. Ozacmak et al (6) previously reported that melatonin treatment attenuated $\mathrm{CCH}$-induced neuropathological alterations in the hippocampus; concordantly, in the present study, the total number of cells in the hippocampal CA1 region of rats in the mel-2VO-group were significantly higher, as compared with in the 2VO-group rats. Therefore, the ability of melatonin to protect against $\mathrm{CCH}$-induced spatial learning and memory impairment may be associated with the neuroprotective effects of melatonin in the hippocampal CA1 region.

Oxidative stress is thought to be a major factor in $\mathrm{CCH}$-induced neuronal cell death $(5,6,28)$. In the present study, the effects of melatonin on $\mathrm{CCH}$-induced oxidative stress in the rat hippocampus were investigated. Melatonin treatment significantly reduced the $\mathrm{CCH}$-induced elevation of MDA levels in the hippocampus of the 2VO-rats, which is consistent with previous studies that reported that the neuroprotective effects of melatonin may be associated with its antioxidant properties in animal models of global and focal cerebral ischemia $(10,14,18)$. Furthermore, these results align with a previous study, in which the protective effects of melatonin against $\mathrm{CCH}$-induced hippocampal neuronal damage were associated with reduced oxidative stress, as indicated by restored levels of MDA, superoxide dismutase and glutathione following melatonin treatment (6).

Morphological and functional microglial alterations are involved in the response to various changes in the neural environment, including ischemic insults $(25,29)$. It was previously reported that $\mathrm{CCH}$ initiates significant microglial activation and neuroinflammatory responses in various regions of the rat brain, including the hippocampus $(1,3,26)$. Furthermore, it has been demonstrated that ischemia- and $\mathrm{CCH}$-induced neuroinflammation promote the expression of pro-inflammatory cytokines, including TNF- $\alpha$ and IL-1 $\beta$, and that these have important roles in the progression of post-ischemic brain injury $(26,30)$. In addition, activated microglia were shown to secrete pro-inflammatory cytokines, including TNF- $\alpha$ and IL-1 $\beta$, in the central nervous system (29). In our previous study, we detected neuroprotective effects for melatonin against ischemic damage, and suggested that these effects may be associated with attenuation of microglial activation in the hippocampal CA1 region following transient global cerebral ischemia (10). Furthermore, previous studies detected attenuation of transient focal cerebral ischemia- and lipopolysaccharide-induced neuroinflammation following melatonin treatment $(20,21)$. In the present study, microglia in the $2 \mathrm{VO}$-group rats were activated and aggregated in proximity to the stratum pyramidale of the ischemic hippocampal CA1 region, whereas this behavior was markedly reduced in the mel-2VO-group rats. In addition, treatment of the $2 \mathrm{VO}$ rats with melatonin significantly decreased the $\mathrm{CCH}$-induced elevation of TNF- $\alpha$ and IL-1 $\beta$ levels in the hippocampus. To the best of our knowledge, the present study is the first to demonstrate an anti-inflammatory effect for melatonin against $\mathrm{CCH}$-induced pro-inflammatory cytokine expression, which suggests that melatonin treatment may attenuate $\mathrm{CCH}$-induced neuroinflammation. Therefore, the results of the present study suggested that the neuroprotective effects of melatonin against $\mathrm{CCH}$-induced neuronal cell damage may be associated with attenuation of microglial activation and neuronal inflammatory responses.

In conclusion, the results of the present study suggested that melatonin was able to improve $\mathrm{CCH}$-induced cognitive impairment, and that the neuroprotective effects of melatonin against $\mathrm{CCH}$-induced hippocampal neuronal cell damage were associated with reduced oxidative stress, attenuation of microglial activation and decreased production of pro-inflammatory cytokines.

\section{Acknowledgements}

The present study was supported by the Basic Science Research Program via the National Research Foundation of Korea, funded by the Ministry of Science, ICT and Future Planning (grant no. NRF-2012R1A1A1007298).

\section{References}

1. Farkas E, Luiten PG and Bari F: Permanent, bilateral common carotid artery occlusion in the rat: A model for chronic cerebral hypoperfusion-related neurodegenerative diseases. Brain Res Rev 54: 162-180, 2007.

2. Román GC: Brain hypoperfusion: A critical factor in vascular dementia. Neurol Res 26: 454-458, 2004.

3. Farkas E, Donka G, de Vos RA, Mihály A, Bari F and Luiten PG: Experimental cerebral hypoperfusion induces white matter injury and microglial activation in the rat brain. Acta Neuropathol 108: 57-64, 2004.

4. He XL, Wang YH, Bi MG and Du GH: Chrysin improves cognitive deficits and brain damage induced by chronic cerebral hypoperfusion in rats. Eur J Pharmacol 680: 41-48, 2012.

5. Kasparová S, Brezová V, Valko M, Horecký J, Mlynárik V,Liptaj T, Vancová O, Ulicná O and Dobrota D: Study of the oxidative stress in a rat model of chronic brain hypoperfusion. Neurochem Int 46: 601-611, 2005.

6. Ozacmak VH, Barut F and Ozacmak HS: Melatonin provides neuroprotection by reducing oxidative stress and HSP70 expression during chronic cerebral hypoperfusion in ovariectomized rats. J Pineal Res 47: 156-163, 2009.

7. Xi Y, Wang M, Zhang W, Bai M, Du Y, Zhang Z, Li Z and Miao J: Neuronal damage, central cholinergic dysfunction and oxidative damage correlate with cognitive deficits in rats with chronic cerebral hypoperfusion. Neurobiol Learn Mem 109: 7-19, 2014.

8. Cechetti F, Pagnussat AS, Worm PV, Elsner VR, Ben J, da Costa MS, Mestriner R, Weis SN and Netto CA: Chronic brain hypoperfusion causes early glial activation and neuronal death, and subsequent long-term memory impairment. Brain Res Bull 87: 109-116, 2012.

9. Feng Z, Lu Y, Wu X, Zhao P, Li J, Peng B, Qian Z and Zhu L: Ligustilide alleviates brain damage and improves cognitive function in rats of chronic cerebral hypoperfusion. J Ethnopharmacol 144: 313-321, 2012.

10. Lee CH, Yoo KY, Choi JH, Park OK, Hwang IK, Kwon YG, Kim YM and Won MH: Melatonin's protective action against ischemic neuronal damage is associated with up-regulation of the MT2 melatonin receptor. J Neurosci Res 88: 2630-2640, 2010.

11. Zhu B, Wang ZG, Ding J, Liu N, Wang DM, Ding LC and Yang C: Chronic lipopolysaccharide exposure induces cognitive dysfunction without affecting BDNF expression in the rat hippocampus. Exp Ther Med 7: 750-754, 2014.

12. De Jong GI, Farkas E, Stienstra CM, Plass JR, Keijser JN, de la Torre JC and Luiten PG: Cerebral hypoperfusion yields capillary damage in the hippocampal CA1 area that correlates with spatial memory impairment. Neuroscience 91: 203-210, 1999. 
13. Sarti C, Pantoni L, Bartolini L and Inzitari D: Cognitive impairment and chronic cerebral hypoperfusion: What can be learned from experimental models. J Neurol Sci 203-204: 263-266, 2002.

14. Reiter RJ, Tan DX, Osuna C and Gitto E: Actions of melatonin in the reduction of oxidative stress. A review. J Biomed Sci 7 : 444-458, 2000.

15. Zhang Y, Li L, Xiang C, Ma Z, Ma T and Zhu S: Protective effect of melatonin against Adriamycin-induced cardiotoxicity. Exp Ther Med 5: 1496-1500, 2013.

16. Ma J, Shaw VE and Mitrofanis J: Does melatonin help save dopaminergic cells in MPTP-treated mice? Parkinsonism Relat Disord 15: 307-314, 2009

17. Reiter RJ, Cabrera J, Sainz RM, Mayo JC, Manchester LC and Tan DX: Melatonin as a pharmacological agent against neuronal loss in experimental models of Huntington's disease, Alzheimer's disease and parkinsonism. Ann NY Acad Sci 890: 471-485, 1999.

18. Lee EJ, Lee MY, Chen HY, Hsu YS, Wu TS, Chen ST and Chang GL: Melatonin attenuates gray and white matter damage in a mouse model of transient focal cerebral ischemia. J Pineal Res 38: 42-52, 2005

19. Letechipía-Vallejo G, López-Loeza E, Espinoza-González V González-Burgos I, Olvera-Cortés ME, Moralí G and Cervantes M: Long-term morphological and functional evaluation of the neuroprotective effects of post-ischemic treatment with melatonin in rats. J Pineal Res 42: 138-146, 2007.

20. Lee MY, Kuan YH, Chen HY, Chen TY, Chen ST, Huang CC, Yang IP, Hsu YS, Wu TS and Lee EJ: Intravenous administration of melatonin reduces the intracerebral cellular inflammatory response following transient focal cerebral ischemia in rats. J Pineal Res 42: 297-309, 2007.

21. Tyagi E, Agrawal R, Nath C and Shukla R: Effect of melatonin on neuroinflammation and acetylcholinesterase activity induced by LPS in rat brain. Eur J Pharmacol 640: 206-210, 2010.
22. Institute of Laboratory Animal Research, Committee for the Update of the Guide for the Care and Use of Laboratory Animals and National Research Council: Guide for the care and use of laboratory animals. 8th edition. National Academies Press, Washington, D.C., USA: p220, 2011.

23. Yoo DY, Kim W, Lee CH, Shin BN, Nam SM, Choi JH, Won MH, Yoon YS and Hwang IK: Melatonin improves D-galactose-induced aging effects on behavior, neurogenesis, and lipid peroxidation in the mouse dentate gyrus via increasing pCREB expression. J Pineal Res 52: 21-28, 2012.

24. Schmued LC and Hopkins KJ: Fluoro-Jade B: A high affinity fluorescent marker for the localization of neuronal degeneration. Brain Res 874: 123-130, 2000.

25. Sugawara T, Lewén A, Noshita N, Gasche Y and Chan PH: Effects of global ischemia duration on neuronal, astroglial, oligodendroglial, and microglial reactions in the vulnerable hippocampal CA1 subregion in rats. J Neurotrauma 19: 85-98, 2002.

26. Qu J, Zhou Q, Du Y, Zhang W, Bai M, Zhang Z, Xi Y, Li Z and Miao J: Rutin protects against cognitive deficits and brain damage in rats with chronic cerebral hypoperfusion. Br J Pharmacol 171: 3702-3715, 2014.

27. Pappas BA, de la Torre JC, Davidson CM, Keyes MT and Fortin T: Chronic reduction of cerebral blood flow in the adult rat: Late-emerging CA1 cell loss and memory dysfunction. Brain Res 708: 50-58, 1996.

28. Li Y, He Y, Guan Q, Liu W, Han H and Nie Z: Disrupted iron metabolism and ensuing oxidative stress may mediate cognitive dysfunction induced by chronic cerebral hypoperfusion. Biol Trace Elem Res 150: 242-248, 2012.

29. Stoll G, Jander S and Schroeter M: Inflammation and glial responses in ischemic brain lesions. Prog Neurobiol 56: 149-171, 1998.

30. Dirnagl U, Iadecola C and Moskowitz MA: Pathobiology of ischaemic stroke: An integrated view. Trends Neurosci 22: 391-397, 1999. 